\title{
ANALYSIS ON CONTINUOUS PARTICIPATION BEHAVIOR OF VIRTUAL COMMUNITY MEMBER BASED ON THE USABILITY AND SOCIABILITY PERSPECTIVE
}

\author{
Kustriyanti Sri*, Astuti Endang Siti, Kusumawati Andriani \\ Department of Business Administration, Faculty of Administrative Science, \\ University of Brawijaya, Indonesia \\ *E-mail: sriversi2009@gmail.com
}

\begin{abstract}
The purpose of this research was to identify some considerable factors that influenced the continuous participation behavior of virtual community member based on the usability and sociability perspective. The type of research conducted was an explanatory research. The data collection technique was to distribute online questionnaire to members of various learning groups on Facebook using snowballing technique. The data obtained from 116 corespondents were analyzed using SEM-PLS (SmartPLS Software). The research findings showed that Perceived Enjoyment did not have significant effect towards Continuous Participation Intention. The finding was different from the findings of studies on Continuous Participation Behavior that applied to leisure-oriented virtual community of which purpose was channeling the members' hobbies; it showed that Perceived Enjoyment had significant influence towards Continuous Participation Intention while Perceived Usefulness did not have significant influence on the Continuous Participation Intention. Based on the result, it may be said that Perceived Usefulness was more important in virtual learning community than Perceived Enjoyment; the opposite result was applicable for the leisure-oriented virtual community. Based on the study, it may be concluded that the Continuous Participation Behavior of its members determined the succees of virtual community. It is important for the founder and virtual community management on Facbeook to write down particul ary the objective and the policy as the guidelines for the comunication and the interaction between virtual community members. It is suggested that users should understand and obey the objective and policy that apply. Common citizen can optimize the use of virtual communities to improve self-quality independently through acceess to knowledge in the learning group.
\end{abstract}

\section{KEY WORDS}

Usability, sociability, virtual community, continuous participation intention, continuous participation behavior.

Virtual community appears as a new phenomena when internet becomes more accessible. Based on ecology-based community development point of view, internet technology has a potential that allows individuals to improve their life quality autodidactly through their self initiative. Virtual community that is formed by individuals who have an intention to improve quality of their lives is one of the information technology application vessels. The life quality improvement can be expressed in the form of culture, health, enviroment and education in which the programme and the ideas comes from individual. Virtual community character is developed using bottom-up approach (innitiative coming from individual). Community being developed by decent citizen usually refers tocommunity empowerment (Ife and Tesoriero, 2008). Community becomes a vessel for a group of people to participate in the improvement of quality of their lives through different kinds of activities that useful for themselves and the environment.

The success of virtual community can be measured through the achievement of a defined goal. Basically, community is categorized as a successful one when there are continuous participation from its members in order to achieve their common goals. Continuous participation in virtual community becomes important since such participation represents participation or willingness of an individual to improve himself or hersepf continuously. Success of information system theory developed by DeLone and McLean is 
widely used widely when information technology application start to be implemented in all aspect of life. The theory may be used to measure success of virtual community that comes with the support of the internet. Some studies (Lin, et al., 2007; Lin, 2007) adapted DeLone and McLean's success of information system theory. Meanwhile, Preece (2001) developed model for measuring virtual community success using the usability and sociability perspective.

Observing participation of virtual community members using the community development point of view that has an objective to improve quality of life based on self initiative through learning proccess is interesting. There is a gap absence in studies that reviews continuous participation of an individual in learning vrtual community that is characterized improvement program based on self initiative and voluntary. Lu et al., (2011) observed the behavior of leisure-oriented virtual community members, while Sun et al., (2012) proved that participation behavior in the form of sharing knowledge was affected by both intrinsic and extrinsic motivation people had when they were joining the virtual community. Phang et al., (2009) examined a virtual community of which members were people who had intention to study in a university. The present study focused on studying the factors that influenced individual continuos participation in the context of virtual community of which objective was to improve quality of life through learning proccess by using Facebook as a means of communication and media of interaction between members. It adopted the usability and sociability perspective as an effort to highlight virtual community from sociotechnic aspect. By using socio-technic perspective, then the basis for behavior understanding was an analysis towards system being applied and various social aspects involved in the communication and interaction. The difference between the current study and the previous ones was selection of virtual community of which objective was self improvement through Facebook as medium for learning proccess. Based on the description, our current research title was "Continuous Participation Behavior of Virtual Community Member based on the Usability and Sociability Perspective". The subject was virtual community member who used Facebook as their technology platform.

\section{LITERATURE REVIEW}

Virtual community is a group of individuals who joins for common purpose. This community interaction and communication is mediated by technology. The definition of virtual community according to Porter (2004) is "an aggregation of individuals or business partners who interact around a shared interest, where the interaction is at lest supported and/or mediated by technology and guided by some protocols or norms". Virtual community becomes a part of cyberspace life. According to Piliang (2004), cyberspace was 'Intermediary world', that was a world of bits of information that was capable of creating many kinds of visual interaction and social relation. 'Intermediary world' explains that relation and social interaction in cyberspace is not physical in a given geographical area. Absence of physical encounter becomes a reason of behavioral change occuring to the individuals, social interaction and community. On the level of individual, cyberspace is capable of transforming the concept of self-identity and people identity.

The utilization of technology for mediatization the communication and interaction brings through the formation of individual behavior. The success virtual of community is strongly influenced by individual behavior of its members. Behavior of members that continuously participate is the veins of virtual community life, and therefore understanding factors that influence the behavior becomes important. From the point of view of usability and sociability then, observation on behavior can be done by considering technology and social factor forming the virtual community.

Usability is the capacity of virtual community that can be used conveniencely and efectively by someone in order to actualize the desired activity. Usability factor can be measured by the quality of information and the system. Quality of information is the character of valuable information that is resulted by the virtual community. System Quality is the character of virtual community performance which provides convenience to the user. These 
two qualities were used as benchmark of virtual community success (Lin et al, 2007; Lin, 2008). Sociability is the character of virtual community which supports social condition where the members can found joy while they are interacting to each other in order to achieve community objective through the space that is enabled by technology. De Souza and Preece (2004) developed a framework of virtual community success by using sociability as one of the components consisting of goals and policies.

Perceived Usefulness is a level of confident where someone believes that using a system will provide an advantage. Gupta and Kim (2007) has tested the functional (system) and social benefit (interaction and communication) to assess the confident of members of virtual community. Perceived enjoyment is level of individuals confident where they can assure that using an information technology will provide a enjoyment. Lu et al. (2011) has proven that enjoyment factor influences the member's intention to keep participating in virtual community. Meanwhile Sun et al. (2012) explained that enjoyment factor was an intrinsic motive that influenced the participation of virtual community member. Sense of belonging is a level of individuals confidence where they can be sure that using a system will bring them a sense of belonging to virtual community. Sense of belonging is obtained through comfort, meaning and commonality of member's vision. Members channel theirwho their sense of belonging in the form of concern, involvement, pride and loyalty to the other members in virtual community.

Continuous participation Intention is a motivational factor when someone has intention to actualize participation behavior. The decision to represent certain behavior is the result of a rational proccess $t$ being directed to a certain objective by following a sequence of thinking (Baron et al., 2003). The definition shows that individual behavior is the result of a sequence of thinking proccess by taking into account many kind of options so that in the end decision he or she makes expresses certain behavior. One of the factors that influences behavior is intention. Someone's intention to participate in the future can be seen by his or her willingness to exchange information and interaction. Continuous Participation Behavior is a sequence of continuous participation repetition. This behavior can be actualized in form the of constantly exchanging information, interaction, and participation. Munnuka et al. (2015) stated that the continuous participation may be seen when the member invited other people and informed other people about the existance of their virtual community.

\section{METHODS OF RESEARCH}

The type of the study was explanatory research with quantitative approach. In the Explanation Research, there is an effort to test the theory by measuring research variable using statistical procedure. The subjects were 116 people from 70 learning communities on Facebook.

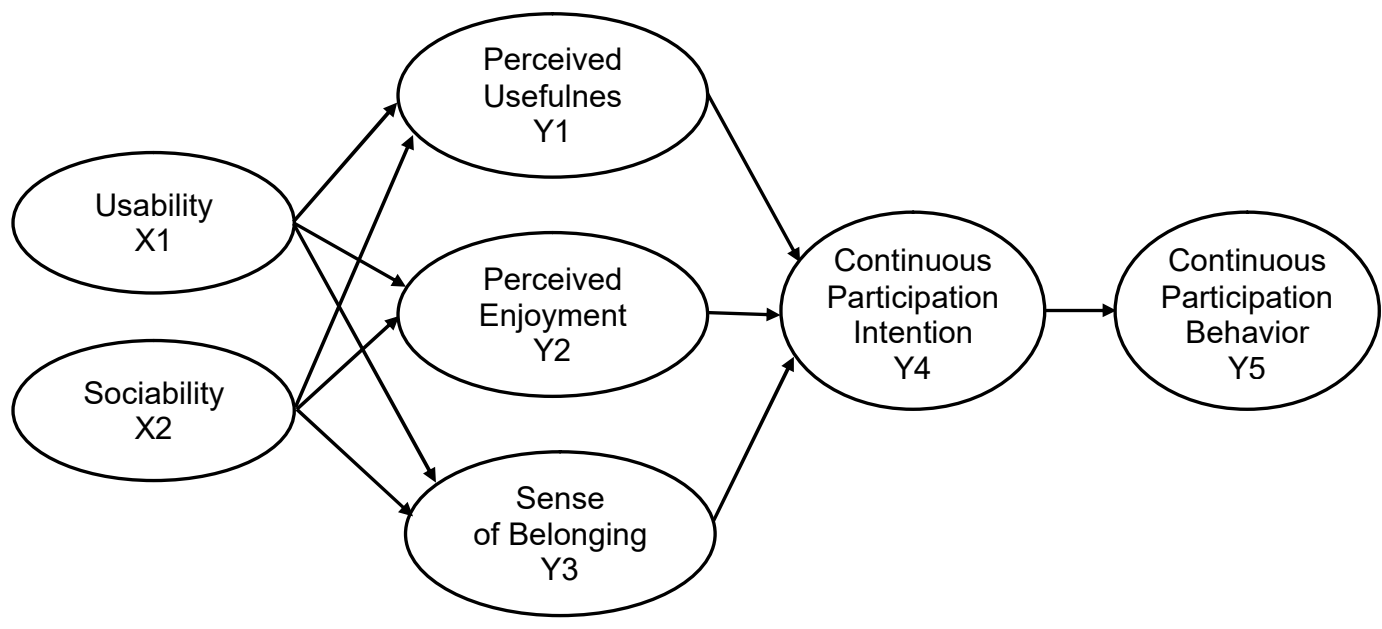

Figure 1 - Conceptual Framework 
This learning community was developed as medium for people interested in learning arts, computer, handicraft, healthy life style, business, gardening, languange, religion and teaching through Facebook.

The research instrument used to obtain the data was online questionnaire distributed the using snowballing method. The method has been used in social media research when online questionnaire was distributed to population of which characters was inaccessible and having low response (Dusek et al., 2015; Kosinski et al., 2016). The data analysis method was SEM-PLS with SmartPLS software. This tool was selected because the general research objective was to test the influnce between variables. Figure 1 described the conceptual framework of the study.

\section{RESULTS AND DISCUSSION}

The purpose of evaluating the model (outer model) was to measure the validity and reliability of variables. The test result showed that the seven variables were considered valid and reliable since their AVE scores were higher than 0.5 and their composite reliability scores were higher than 0.7 .

Structural model testing (Inner Model) aimed at measuring the correlation between variables. There were some measurement of SmartPLS that can be used to test structural model. In the study, the model fit would be used to see how good the submitted structural model was. To see that, the researcher used the Standardized Root Mean Square Residual (SRMR) which tested the difference between observed correlation and cultivated correlation in matrix correlation. Basically, SRMR calculated the magnitude between correlation. SRMR value cwas onsidered good if $<10$. Based on Tabel 5 . The SRMR of the model was $0.082<$ 10 , and thus, the model was considered fit.

Table 1 - Fit Model

\begin{tabular}{|c|c|}
\hline $\mathrm{n} / \mathrm{n}$ & Saturated Model \\
\hline SRMR & 0.082 \\
\hline NFI & 0.606 \\
\hline Rms Theta & 0.298 \\
\hline
\end{tabular}

The second model fit test was the Normed Fit Index (NFI) or Fit Index. NFI score is between 0 and 1; when NFI score of a model is closer to 1 , the model fit is better. The NFI of the structural model was 0.606 and thus, the model was considered fit. The third model fit test was the rms Theta that used for reflective model. Rms Theta score is between 0 to 1; when the rms Theta score is closer to 0 , the model fit is better. Basically, rms Theta compare observed indicator and predicted indicator. The rms Theta score was 0.298 and therefore, the model was considered fit.

Table 2 - Path Coefficient

\begin{tabular}{|c|c|c|c|c|c|c|}
\hline $\mathrm{n} / \mathrm{n}$ & $\begin{array}{c}\text { Original } \\
\text { Sample (O) }\end{array}$ & $\begin{array}{c}\text { Sample } \\
\text { Mean (M) }\end{array}$ & $\begin{array}{c}\text { Standard } \\
\text { Deviation } \\
(\text { STDEV) }\end{array}$ & $\begin{array}{c}\text { T Statistics } \\
(|\mathrm{O} / \mathrm{STDEV}|)\end{array}$ & $\mathrm{P}$ Values & Description \\
\hline CPI -> CPB & 0.765 & 0.765 & 0.046 & 16.556 & 0.000 & Significant \\
\hline PE -> CPI & 0.032 & 0.041 & 0.115 & 0.281 & 0.779 & Insignificant \\
\hline PU -> CPI & 0.217 & 0.210 & 0.101 & 2.147 & 0.032 & Significant \\
\hline SoB -> CPI & 0.517 & 0.516 & 0.109 & 4.724 & 0.000 & Significant \\
\hline Soc -> PE & 0.401 & 0.403 & 0.088 & 4.571 & 0.000 & Significant \\
\hline Soc -> PU & 0.411 & 0.410 & 0.095 & 4.347 & 0.000 & Significant \\
\hline Soc -> SoB & 0.260 & 0.264 & 0.111 & 2.329 & 0.020 & Significant \\
\hline Usa -> PE & 0.259 & 0.268 & 0.094 & 2.751 & 0.006 & Significant \\
\hline Usa -> PU & 0.274 & 0.284 & 0.093 & 2.948 & 0.003 & Significant \\
\hline Usa -> SoB & 0.414 & 0.416 & 0.112 & 3.685 & 0.000 & Significant \\
\hline
\end{tabular}


Having finished evaluating the model fit, the following procedure was to conduct hypothesis testing. The purpose of the hypothesis testing was to describe the relationship between variables. There were ten hypothesis that submitted in the study, and the basis for discussing each of them was the Path Coefficient. The table below mentioned the parameter of the Original sample which showed whether a correlation had either positive influece or negative influence. The next measurement for the level of significance was T-Statistics. The level of the significance in the study was $5 \%$ and the t-value was $>1.96$ (two-tailed). The level of significance may also be observed based on $p$-value; one variable has significant influence when the $p$-value was $<0.05$.

Based on Table 2, it can be seen that out of the the ten hypotheses, nine of them had significant influence but one of them, the Perceived Enjoyment to Continuous Participation Intention (path coefficient of 0.032 , t-statistics of 0.281 and p-value of 0.0779 ) did not. It is different from the finding of the study conducted by Lu et al., (2011) of which subjects were the leisure-oriented virtual community. Lu, et.al (2011)'s study described that Perceived Enjoyment had significant influence towards Continuous Participation Intention meanwhile Perceived Usefulness did not have significant influence towards the Continuous Participation Intention. The difference between these findings shows that different behavior occurs when the objective that settled by virtual community is also different. In the virtual community of which objective is learning, the Perceived Usefulness is an important factor that influences someone's intention to continuously participating meanwhile Perceived Enjoyment is not.

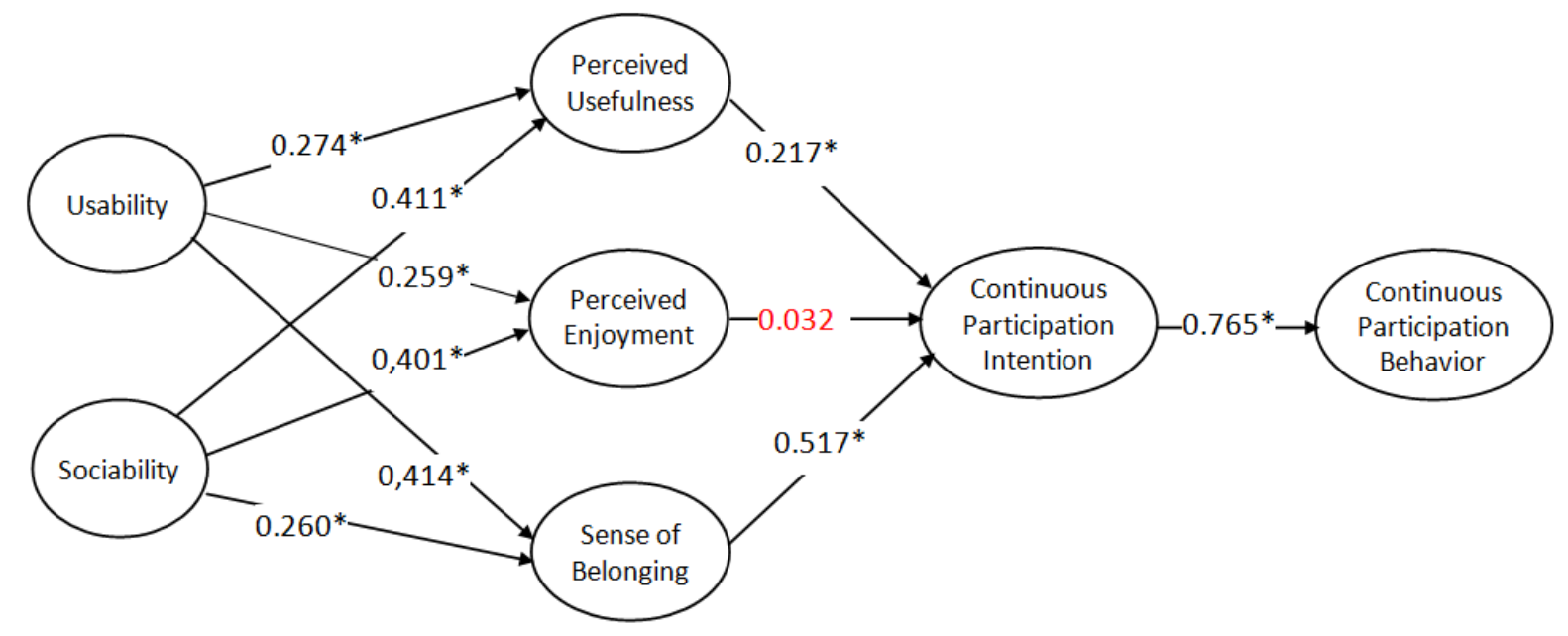

*have a significant effect on the level of $\alpha=5 \%$

Figure 2 - Hypothesis testing

This finding also showed that Usability and Sociability had significant influence towards the Perceived Usefulness, Perceived Enjoyment and Sense of Belonging. Sense of Belonging had significant influence towards Continuous Participation Intention. It is in line with the previous studies conducted by Lu et al. (2011), Lin et al. (2007), Lin (2007) and Lin (2008). Continuous Paticipation Intention had significant influence towards Continuous Participation Behavior, which is in accordance to a study conducted by Al-Debei et al. (2013) and Wang et al. (2015). It means that Continuous Participation Behavior of virtual community members, observed from the Usability and Sociability point of view, is influenced by the driven intention that comes from the Perceived Usefulness and sense of Belonging.

\section{CONCLUSION}

The study attempts at explaining the factors which influence the Continuous Participation Behavior of virtual community based on the Usability and Sociability perspectives. The researchers are interested in studying Continuous Participation Behavior 
because it is an important factor in the success of a virtual community. The current research findings show that nine hypotheses are proven to have significant influence while one hypothesis does not. In the learning-oriented virtual community context, Perceived Usefulness has been proven as an important factor. Meanwhile, for a leisure-oriented virtual community, Perceived Enjoyment is the variable that influences Continuous Paticipation Intention. In the learning-oriented virtual community, the members focus more on gaining information, increasing knowledge, insight and getting solution for problems that they experience. Generally, some people are motivated to keep participating in virtual community when Usability aspect and sociability aspect can be fulfilled.

\section{LIMITATIONS AND SUGGESTIONS}

There are some limitations in the study that readers may take into account in evaluating the findings. First, the study portrays one phenomenon (continuous participation behavior) in one short interval of time. As the consequence, the researchers may not be able to conclude the dynamic of individual behavior. As an addition, it does not observe the routines of the virtual community members. In terjms of Continuous participation, it is important to describe the relationship between the pattern of behavior and membership duration. Based on the explanation above, future research should conduct studies on the same topic using longitudinal approach in order to potray dynamical behavior of continuous participation at certain interval of time.

The second, the study does not involve 'Trust' as variable to evaluate how much an individual believes information being exchanged in virtual community. Previous research (Hsu et al., 2007 and Chiu et al., 2006) involved 'trust' to describe the phenomena. In the learning context developed by the publicthat formed by common citizen, the conventional teacher-student role does not take place during learning proccess that occurs in virtual community. The source of information is an individual who voluntary shares his or her knowledge. There is no guarantee that particulare piece of information shared on virtual community is valid. Therefore, future researcher should conduct studies that compare learning virtual community developed by the society and one developed by certain educational institution.

\section{REFERENCES}

1. Al-Debei, M. Mutaz, Enas Al-Lozi, Anastasia Papazafeiropoulou. 2013. Why people keep coming back to facebook: explaining and predicting continuance participation from an extended theory of planned behavior perspective. Decision Support Systems, 55:43-54.

2. Baron, A. Robert \& Donn Byrne. 2003. Psikologi Sosial. Translated by R. Djuwita. Penerbit Erlangga. Jakarta.

3. Chiu Chiu, Chao-Min, Meng-Hsiang Hsu, Eric T.G. Wang. 2006. Understanding knowledge sharing in virtual communities: an integration of social capital and social cognitive theories. Decision Support System 42:1872-1888.

4. De-Souza, Clarisse Sieckenius, Jenny Preece. 2004. A framework for analyzing and understanding online communities. The Interdisciplinary Journal of Human-Computer Interaction.

5. Dusek, Gery A, Yuliya V. Yurova, Cynthia P. Rupperl. 2015. Using Social Media and Targeted Snowball Sampling to Survey a Hard-to-reach Population: A Case Study. International Journal of Doctoral Studies 10, 279-299.

6. Ife, J \& F. Tesoriero. 2008. Community Development : Alternatif Pengembangan Masyarakat di Era Globalisasi. Translated by S. Manulang. Pustaka Pelajar Yogyakarta.

7. Hsu, Meng-Hsiang, Teresa L. Ju, Chia Hui Yen, Chun-Ming Chang. 2007. Knowledge sharing behavior in virtual communities: the relationship between trust, self-efficacy, and outcome expectations. Human Computer Studies 65:153-169.

8. Kosinski, Michal, Sandra C. Matz, Samual D. Gosling, Vesselin Popov, David Stillwell. 2016. Facebook as a research tool. Monitor on Psychology. 70-75. 
9. Lin, Hsiu-Fen. 2007. The role of online and offline features in sustaining virtual communities: an empirical study. Internet Research 17(2):119-138.

10. Lin, Hsiu-Fen. 2008. Determinant of succesfull virtual community: Contribution from system characteristics and social factors. Information dan Management 45(8):522-527.

11. Lin, Hui, Weiguo Fan, Linda Wallace. 2007. An empirical study of web-based knowledge community success. IEEE Proceeding of the 40th Annual Hawaii International Conference on System Science.

12. Lu, Xianghua, Chee Wei Phang, Jie Yu. 2011. Encouraging participation in virtual communities through usability and sociability development: an empirical investigation. The Data Base for Advances in Information Systems 42(3): 96-114.

13. Munnuka, Juha, Heikki Karjaluoto, Anna Tikkanen. 2015. Are facebook brand community members truly loyal to the brand? Computers in Human Behaviour 51:429-439.

14. Phang, Chee Wei, Atrevi Kankanhalli, Rajiv Sabherwal. 2009. Usability and sociability in online communities: a comparative study of knowledge seeking and contribution. Journal of the Association for Information Systems 10(10): 721-747.

15. Piliang, A. Yasraf. 2004. Dunia yang Dilipat. Jalasutra. Bandung

16. Porter, CE. 2004. A typology of virtual communities: a multidisciplinary foundation for future research. Journal of Computer-Mediated Communication 10(1).

17. Preece, Jenny. 2001. Sociability and usability in online communities : determining and measuring succes. Behaviour and Information Technnology Journal 20(5):347-356.

18. Sun, Yongqiang, Yulin Fang, Kai H. Lim 2012. Understanding sustained participation in transactional virtual communities. Decision Support Systems 53:12-22. 